Articulo original

(Original paper)

\title{
HERPETOFAUNA DEL SITIO ARQUEOLÓGICO IGLESIA VIEJA, COSTA DE CHIAPAS, MÉXICO
}

\section{HERPETOFAUNA FROM THE ARCHAEOLOGICAL SITE IGLESIA VIEJA, COAST OF CHIAPAS, MEXICO}

\author{
JESÚS M. LÓPEZ-VILA ${ }^{1}$, ARTURO TORRES-MEZA ${ }^{1}$, EMILIO I. ROMERO-BERNY ${ }^{1}$ *, DANIEL PINEDA- \\ VERA $^{2}$ \\ ${ }^{1}$ Centro de Investigaciones Costeras. Instituto de Ciencias Biológicas. Universidad de Ciencias y Artes de Chiapas, Juan José \\ Calzada y Prolongación Calzada de Guadalupe, Col. Evolución, Tonalá, 30500, Chiapas, México; <jesus.lopez@unicach.mx>; \\ <atm_and1@hotmail.com>; <emilio.romero.ex@unicach.mx>; \\ ${ }^{2}$ Programa educativo de Licenciatura en Biología. Instituto de Ciencias Biológicas. Universidad de Ciencias y Artes de Chiapas, \\ Libramiento Norte Poniente N. 1150, Col. Lajas de Maciel, Tuxtla Gutiérrez, 29039, Chiapas, México; \\ $<$ danielp.abronia@gmail.com> \\ *Autor de correspondencia: <emilio.romero.ex@unicach.mx>
}

Recibido: 26/02/2018; aceptado: 22/08/2018; publicado en línea: 16/11/2018

Editor responsable: Gustavo Aguirre

López-Vila, J. M., Torres-Meza, A., Romero-Berny, E. I., Pineda-Vera, D. (2018) Herpetofauna del sitio arqueológico Iglesia Vieja, Costa de Chiapas, México. Acta Zoológica Mexicana (nueva serie) 34, 1-14. https://doi.org/10.21829/azm.2018.3412135

RESUMEN. Se realizó un listado de las especies de anfibios y reptiles del sitio arqueológico Iglesia Vieja. Este sitio se localiza entre la costa y la Sierra Madre de Chiapas a $711 \mathrm{msnm}$. Los muestreos se realizaron mensualmente entre junio de 2014 y mayo de 2015 abarcando las temporadas de lluvias y secas en dos tipos de vegetación (herbácea y arbórea). La herpetofauna estuvo representada por 61 especies (12 anfibios y 49 reptiles). Los anfibios se agruparon en ocho familias, 10 géneros y 12 especies; para los reptiles, el grupo de las lagartijas se constituyó por 13 familias, 16 géneros y 20 especies; mientras que las serpientes estuvieron representadas por siete familias, 25 géneros y 29 especies. Los estimadores de riqueza Jackknife 1 y Bootstrap mostraron una adecuada representación de la composición. Las curvas de abundancia reflejaron una mayor dominancia de especies generalistas o asociadas a perturbación (Hemidactylus frenatus, Basiliscus vittatus, Rhinella horribilis). El 35\% de las especies registradas se encuentra bajo alguna categoría de riesgo de acuerdo a la normativa federal. Aunque el área se localiza en una zona de transición fisiográfica, la herpetofauna mostró una alta afinidad con la Planicie costera del Pacífico (66.53\%). Las áreas destinadas a preservar el patrimonio arqueológico constituyen también espacios que contribuyen a mantener los procesos biológicos. Estos espacios rara vez cuentan con inventarios faunísticos. Este trabajo representa una contribución al conocimiento de la biota asociada a sitios arqueológicos en Chiapas, y el inicio de una línea base para el manejo de una zona arqueológica y su biodiversidad, así como para futuras investigaciones sobre la ecología de un grupo prioritario.

Palabras clave: Riqueza, composición, dominancia, anfibios y reptiles, zona arqueológica, conservación. 
López-Vila, J. M., Torres-Meza, A., Romero-Berny, E. I., Pineda-Vera, D. (2018) Herpetofauna from the archaeological site Iglesia Vieja, Coast of Chiapas, Mexico. Acta Zoológica Mexicana (nueva serie) 34, 1-14. https://doi.org/10.21829/azm.2018.3412135

ABSTRACT. A checklist of amphibians and reptiles in the archaeological site Iglesia Vieja was made. This site is located between the coast and the Sierra Madre of Chiapas at 711 masl. The samplings were carried out monthly between June 2014 and May 2015, covering the rainy and dry seasons in two types of vegetation (herbaceous and forested). The herpetofauna was represented by 61 species (12 amphibians and 49 reptiles). The amphibians were grouped into eight families, 10 genera and 12 species; for reptiles, the group of lizards was constituted by 13 families, 16 genera and 20 species; while snakes were represented by seven families, 25 genera and 29 species. The Jackknife 1 and Bootstrap richness estimators showed an adequate representation of the composition. The abundance curves showed a greater dominance of generalist or disturbance-associated species (Hemidactylus frenatus, Basiliscus vittatus, Rhinella horribilis). Thirty-five percent of the registered species is under some risk category according to federal regulations. Although the area is located in a physiographic transition zone, the herpetofauna showed a high affinity with the Pacific Coastal Plain (66.53\%). The areas destined to preserve the archaeological heritage also constitute spaces that contribute to maintain the biological processes. These sites rarely have faunal inventories. This work represents a contribution to the knowledge of the associated biota with archaeological sites in Chiapas, and groundwork for the management and future research on the ecology of a priority group.

Key words: Richness, composition, dominance, amphibians and reptiles, archaeological site, conservation.

\section{INTRODUCCIÓN}

La herpetofauna del estado de Chiapas destaca como una de las más ricas del país con 330 especies registradas (Johnson et al., 2015a). Al tratarse de la principal zona de transición del norte Mesoamericano, su biota herpetológica incluye numerosas especies que comparte con el núcleo de América Central (Johnson, 1989; Lee, 1996). En los últimos años se ha incrementado notablemente el conocimiento sobre los anfibios y reptiles de Chiapas, no obstante persiste un desconocimiento generalizado para muchas localidades con potencial para el manejo. Así mismo los rasgos fisiográficos estatales conforman patrones de distribución complejos que necesitan estudiarse desde un punto de vista biogeográfico.

Las zonas arqueológicas y los ecosistemas adyacentes a éstas han demostrado ser ambientes adecuados para la conservación de la biodiversidad (Somaweera et al., 2001; Báez et al., 2016). Para el caso específico de la herpetofauna, se ha determinado cierta relación positiva entre el refugio que proveen los sitios arqueológicos y el mantenimiento de algunas poblaciones de especies (Kelly-Hernández et al., 2014; Márquez-Rodríguez, 2014; Olivera et al., 2016).

México cuenta con 187 sitios arqueológicos inventariados por el Instituto Nacional de Antropología e Historia, de los cuales 10 se encuentran en el estado de Chiapas (INAH, 2017). A diferencia de lo que ocurre con las Áreas Naturales Protegidas, los sitios arqueológicos en el país rara vez cuentan con listados faunísticos y planes de manejo que regulen su papel como zonas de protección de la diversidad biológica, aunque la mayoría de estos espacios destinados a preservar el patrimonio cultural son zonificados dentro de un polígono en el que suele mantenerse a los ecosistemas originales. Considerando el elevado número de zonas arqueológicas mexicanas, son limitados los trabajos realizados para conocer a las especies de anfibios y reptiles asociados a estos espacios (Ferreira-García \& CansecoMárquez, 2006; Calderón-Mandujano et al., 2008; CONANP, 2010a; 2010b; Meave del Castillo, 2010; Kelly-Hernández et al., 2014; Mireles-Merchant et al., 2014). 
El sitio de Iglesia Vieja o Tonatlán es uno de los pocos y más importantes vestigios de la cultura Mixe-Zoque en la costa de Chiapas. En los últimos años ha habido un interés de ofertar esta zona como punto de una ruta turística regional, lo cual ha implicado una mayor afluencia de visitantes y el incremento de excavaciones con fines arqueológicos (Kaneko, 2011). Debido a esto es recomendable contar con una línea base referente a la riqueza biológica que permita planificar adecuadamente el manejo del área.

Este estudio aporta información básica sobre la estructura y composición de las comunidades de anfibios y reptiles, comparando la dominancia de las principales especies en dos coberturas de vegetación. Así mismo, la lista taxonómica permite clasificar a este sitio de transición fisiográfica, contribuyendo al conocimiento de los patrones de distribución herpetofaunística a una escala regional. Se espera promover futuras investigaciones que puedan fomentar la conservación de estos organismos en el área de estudio y en consecuencia otorgarle una mayor relevancia a la región.

\section{MATERIALES Y MÉTODOS}

Área de estudio. El sitio arqueológico Iglesia Vieja (SAIV) cuenta con una extensión aproximada de 60 ha

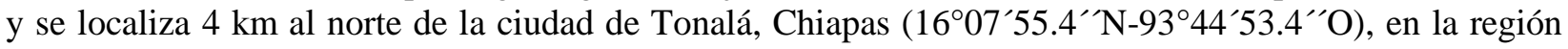
fisiográfica de la Planicie Costera del Pacífico y las estribaciones de la Sierra Madre, a una altitud de 711 msnm (Fig. 1). Presenta un clima cálido-subhúmedo con marcada estacionalidad (lluvias: mayo-octubre; estiaje: noviembre-abril), precipitación anual de $1650 \mathrm{~mm}$ y temperatura media de $27.6^{\circ} \mathrm{C}$ (Kaneko, 2006; INEGI, 2014). La vegetación del área se conforma por remanentes de selva mediana subcaducifolia, sabanas y pastizales inducidos por actividades agropecuarias (Pennington \& Sarukhán, 2005).

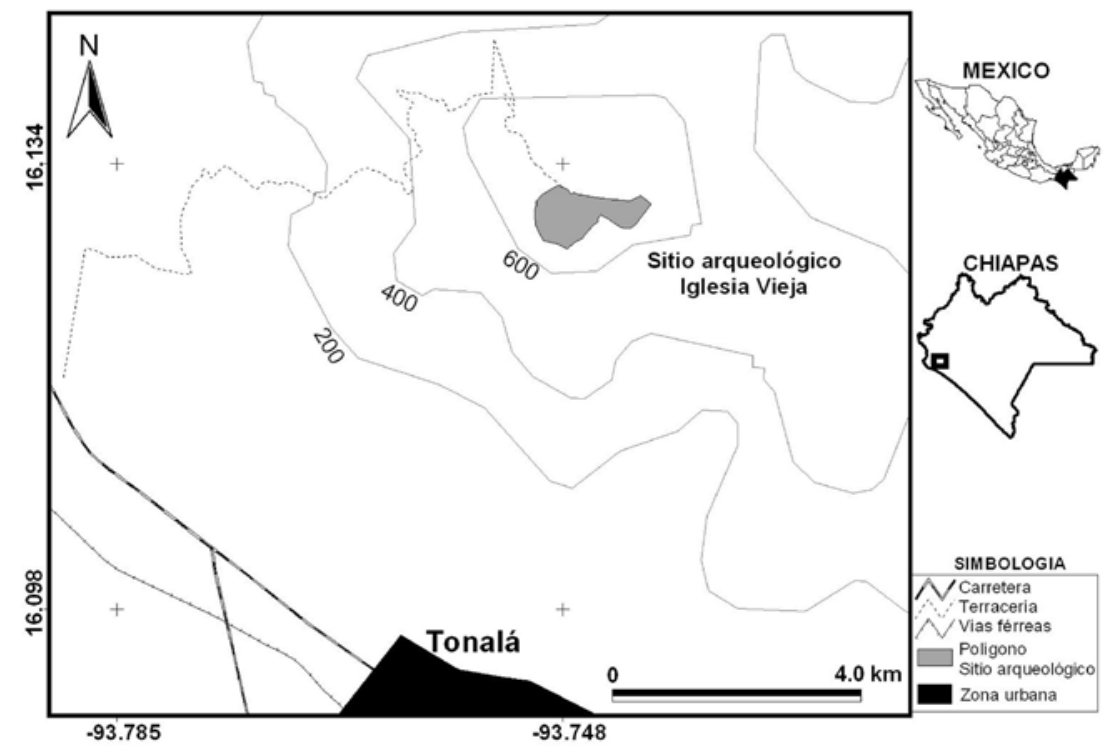

Figura 1. Localización del Sitio Arqueológico Iglesia Vieja, Chiapas.

Muestreo. Los muestreos contemplaron un ciclo anual (entre junio de 2014 y mayo de 2015) y se realizaron de manera mensual con una duración de ocho días cada uno (96 días en total). Así mismo se realizaron visitas adicionales durante los meses de abril, mayo, agosto y octubre de 2016 y 2017 con el fin de verificar algunos registros. La búsqueda de organismos fue al azar mediante la técnica de registros visuales con tiempo definido (Heyer et al., 1994; Lips et al., 2001). Los muestreos por jornada fueron diurnos (9:00-13:00 hrs), vespertinos (17:00-19:00 hrs) y nocturnos (21:00-23:00 hrs), con un esfuerzo de 
tres personas para los horarios establecidos. Se consideraron dos coberturas de vegetación para el muestreo: vegetación herbácea (incluyendo sabanas naturales y pastizales inducidos) y vegetación arbórea (incluyendo acahuales y remanentes de selva con árboles de entre 5 y $20 \mathrm{~m}$ de altura). En cada recorrido se buscaron organismos en microhábitats, tales como debajo de rocas y troncos, en grietas, charcas, entre la hojarasca y el follaje de la vegetación; así como en oquedades y sobre los montículos arqueológicos. La captura de lagartijas y anfibios fue manual, mientras que para las serpientes se utilizó un gancho herpetológico. Los organismos fueron fotografiados e identificados in situ a nivel de especie, para posteriormente ser liberados en el lugar de captura. La determinación taxonómica se realizó con las claves de Flores-Villela et al. (1995) y las guías de Köhler (2008; 2011). Los datos característicos biométricos, obtenidos de los ejemplares en campo, fueron corroborados con los de los organismos depositados en la Colección Herpetológica del Museo de Zoología de la Universidad de Ciencias y Artes de Chiapas (MZUNICACH). Los registros fotográficos fueron catalogados en la plataforma en línea NaturaLista (https://www.naturalista.mx/guides/8109).

Análisis de datos. La lista de especies se elaboró siguiendo la nomenclatura actualizada y arreglo sistemático de acuerdo a Johnson et al. (2015a; 2015b). La representatividad del muestreo para los grupos de anfibios, lagartijas y serpientes, se analizó con los estimadores Jackknife 1 y Bootstrap. El primero es recomendado cuando no se asume homogeneidad ambiental en el muestreo (Percino-Daniel et al., 2013); mientras que el segundo se caracteriza por ser poco sensible al número de especies raras (Moreno, 2001; Magurran, 2004). El número esperado de especies $\left[\mathrm{E}_{(\mathrm{S})}\right]$ para cada grupo de herpetozoos fue analizado duplicando $(n=192)$ y triplicando $(n=288)$ el número de muestras obtenidas para extrapolar a valores de riqueza teórica (Colwell et al., 2005).

El número de especies abundantes por grupo fue visualizado mediante curvas de diversidad acumulada, utilizando el número de primer orden de la serie de Hill $\left(\mathrm{N}_{1}\right)$, calculado a partir del exponencial de la entropía de Shannon (Jost, 2006). Se construyeron curvas de rango-abundancia (con transformación $\log _{10}$ ) de especies. Estas representaciones gráficas de la dominancia constituyen una alternativa robusta para describir los patrones estructurales de una comunidad (McGill et al., 2007). Las pendientes de las curvas resultantes por cobertura vegetal fueron comparadas con un análisis U de MannWhitney, después de haber detectado heterocedasticidad en los datos mediante una prueba de Kolmogorov-Smirnov (Sokal \& Rohlf, 1981).

Para representar jerárquicamente el patrón de similitud entre la herpetofauna del SAIV y la reportada por Johnson et al. (2015a) en siete regiones fisiográficas de Chiapas, se construyó un dendrograma UPGMA utilizando el coeficiente de Kulczynski como medida de disimilitud, basado en una matriz de presencia/ausencia de especies. El coeficiente de Kulczynski recientemente se ha aplicado en estudios biogeográficos como alternativa al de Jaccard, al ser más apropiado para comparar áreas con marcadas diferencias en sus valores de riqueza específica (Hausdorf, 2002; Casazza et al., 2008). Los análisis estadísticos se realizaron utilizando los paquetes Past 3.16 (Hammer et al., 2001) y Primer 6 (Clarke \& Gorley, 2006).

La lista sistemática fue anotada con el estado de conservación de las especies de acuerdo a los criterios de la NOM-059-SEMARNAT (SEMARNAT, 2010), la lista roja de IUCN (IUCN, 2016), CITES y su categoría de vulnerabilidad ambiental (EVS por sus siglas en inglés) propuesta por Wilson et al. (2013a; 2013b).

\section{RESULTADOS}

La herpetofauna del SAIV estuvo conformada por 61 especies, de las cuales 12 fueron de anfibios y 49 de reptiles (Cuadro 1). Los anfibios se agruparon en ocho familias y 10 géneros, mientras que las lagartijas 
constituyeron 13 familias, 17 géneros y 20 especies; por su parte las serpientes estuvieron representadas por 7 familias, 25 géneros y 29 especies. De manera general las familias mejor representadas, tomando en cuenta el número de especies que las constituyen, fueron Colubridae con 13, seguida de Dipsadidae con ocho. De acuerdo al tipo de vegetación, 43 especies fueron registradas en pastizal, mientras que en la vegetación arbórea se registraron 56.
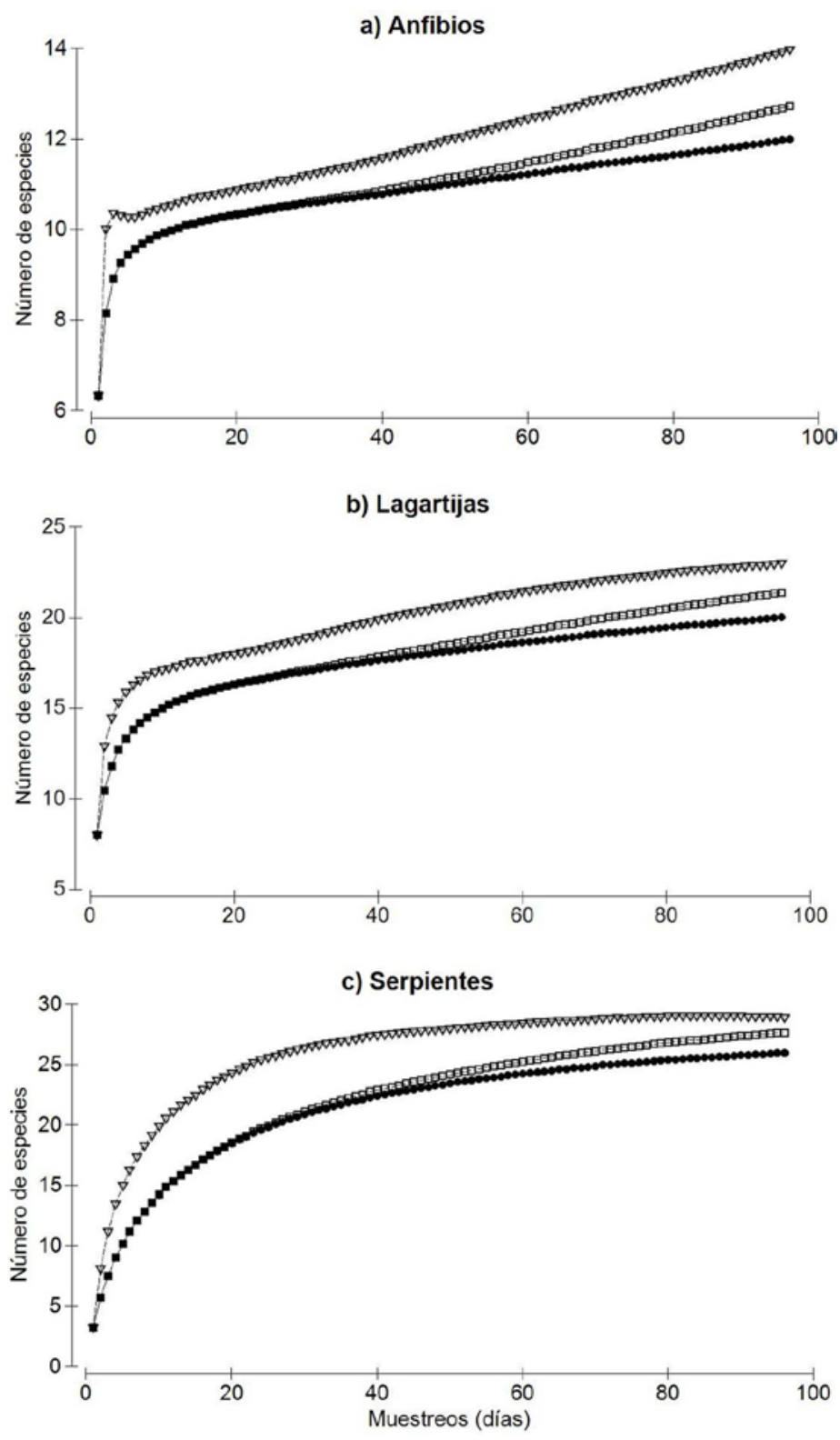

Figura 2. Curvas de acumulación de especies de anfibios, lagartijas y serpientes en el sitio arqueológico Iglesia Vieja. O: Especies observadas, $\nabla$ : Estimador Jackknife 1, $\square$ : Estimador Bootstrap. 
López-Vila et al.: Herpetofauna del sitio arqueológico Iglesia Vieja, Chis.

Cuadro 1. Lista de especies de anfibios y reptiles del sitio arqueológico Iglesia Vieja, Tonalá, Chiapas, México. Estado de conservación de acuerdo a la NOM-059-SEMARNAT-2010: (PR) Sujeta a protección especial, (A) Amenazada; Lista Roja de Especies Amenazadas (IUCN, 2015): (LC) Least Concern, (NT) Near Threatened, (VU) Vulnerable; Environmental Vulnerability Score (EVS) (Johnson et al., 2015a): (B) Baja, (M) Media, (A) Alta. Se indica la presencia de especies por tipo de cobertura vegetal.* Especie exótica.

Taxa

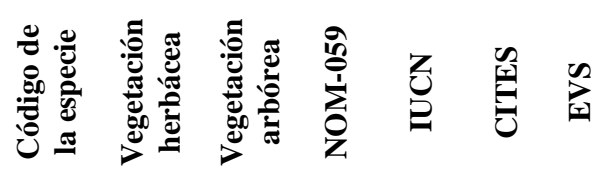

\section{CLASE AMPHIBIA}

ORDEN ANURA

FAMILIA BUFONIDAE

Incilius canaliferus (Cope, 1877)

Incilius coccifer (Cope, 1866)

Incilius marmoreus (Wiegmann, 1833)

Rhinella horribilis (Linnaeus, 1758)

FAMILIA CRAUGASTORIDAE Craugastor loki (Shannon \& Werler, 1955)

FAMILIA ELEUTHERODACTYLIDAE

Eleutherodactylus pipilans Taylor, 1940

FAMILIA HYLIDAE

Smilisca baudinii (Duméril \& Bibrón, 1841)

Trachycephalus typhonius (Linnaeus, 1758)

FAMILIA LEPTODACTYLIDAE Leptodactylus melanonotus (Hallowell, 1861)

FAMILIA MICROHYLIDAE

Hypopachus ustus (Coper, 1866)

FAMILIA RANIDAE

Lithobates forreri (Boulenger, 1883)

ORDEN GYMNOPHIONA

FAMILIA DERMOPHIIDAE

Dermophis mexicanus (Duméril \& Bibrón, 1841)

\section{CLASE REPTILIA}

ORDEN SQUAMATA

FAMILIA ANGUIDAE

Gerrhonotus liocephalus Wiegmann, 1828

FAMILIA CORYTOPHANIDAE

Basiliscus vittatus Regan, 1909

FAMILIA DACTYLOIDAE

Norops serranoi (Köhler, 1999)

Norops unilobatus (Kölher \& Vesely, 2010)

FAMILIA EUBLEPHARIDAE

Coleonyx elegans Gray, 1845

FAMILIA GEKKONIDAE

Hemidactylus frenatus (Duméril \& Bibrón, 1836)*

FAMILIA HELODERMATIDAE

Heloderma horridum (Wiegmann, 1829)

FAMILIA IGUANIDAE

Ctenosaura pectinata (Wiegmann, 1834)

Ctenosaura similis (Gray, 1830)

Iguana iguana (Linnaeus, 1758)

$\begin{array}{llllll}\text { Ica } & \text { X } & \text { X } & & \text { LC } & \text { B } \\ \text { Ico } & \text { X } & \text { X } & \text { Pr } & \text { LC } & \text { B } \\ \text { Im } & \text { X } & \text { X } & & \text { LC } & \text { M } \\ \text { Rh } & \text { X } & \text { X } & & \text { LC } & \text { B } \\ \text { Cl } & \text { X } & \text { X } & & \text { LC } & \text { M } \\ \text { Ep } & \text { X } & \text { X } & & \text { LC } & \text { M } \\ \text { Sb } & \text { X } & \text { X } & & \text { LC } & \text { B } \\ \text { Tt } & \text { X } & \text { X } & & \text { LC } & \text { B } \\ \text { Lm } & \text { X } & \text { X } & & \text { LC } & \text { B } \\ \text { Hu } & & \text { X } & \text { Pr } & \text { LC } & \text { B } \\ & & & & & \\ \text { Lf } & \text { X } & \text { X } & \text { Pr } & \text { LC } & \text { B } \\ & & & & & \\ \text { Dm } & & \text { X } & \text { Pr } & \text { VU } & \text { M }\end{array}$

FAMILIA PHRYNOSTOMIDAE

Sceloporus melanorhinus Bocourt, 1876

\begin{tabular}{|c|c|c|c|c|c|}
\hline Gl & & $\mathrm{X}$ & $\operatorname{Pr}$ & LC & \\
\hline $\mathrm{Bv}$ & $X$ & $\mathrm{X}$ & & LC & \\
\hline Ns & & $X$ & & & \\
\hline $\mathrm{Nu}$ & $X$ & $\mathrm{X}$ & & & \\
\hline Ce & $X$ & $X$ & A & LC & \\
\hline $\mathrm{Hf}$ & $X$ & $\mathrm{X}$ & & LC & \\
\hline Hh & $X$ & & A & LC & II \\
\hline Сp & $X$ & $\mathrm{X}$ & A & & \\
\hline Cs & $\mathrm{X}$ & $\mathrm{X}$ & A & LC & \\
\hline Ii & & $X$ & $\mathrm{Pr}$ & & II \\
\hline Sm & $X$ & $\mathrm{X}$ & & LC & \\
\hline
\end{tabular}




\begin{tabular}{|c|c|c|c|c|c|c|c|}
\hline Taxa & 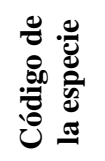 & 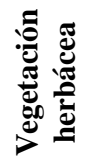 & 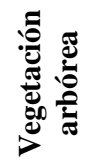 & 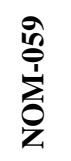 & $\begin{array}{l}Z \\
\text { Z }\end{array}$ & 䛼 & $\sum_{i=1}^{\infty}$ \\
\hline Sceloporus siniferus Cope, 1869 & Ss & $\mathrm{X}$ & $\mathrm{X}$ & & LC & & $\mathrm{M}$ \\
\hline Sceloporus variabilis Wiegmann, 1834 & Sv & $\mathrm{X}$ & $\mathrm{X}$ & & LC & & B \\
\hline Urosaurus bicarinatus (Duméril, 1856) & Ub & $\mathrm{X}$ & $\mathrm{X}$ & & $\mathrm{LC}$ & & M \\
\hline \multicolumn{8}{|l|}{ FAMILIA PHYLLODACTYLIDAE } \\
\hline Phyllodactylus tuberculosus Wiegmann, 1834 & $\mathrm{Pt}$ & $\mathrm{X}$ & $\mathrm{X}$ & & LC & & B \\
\hline \multicolumn{8}{|l|}{ FAMILIA SPHAERODACTYLIDAE } \\
\hline $\begin{array}{l}\text { Sphaerodactylus glaucus (Cope, 1866) } \\
\text { FAMILIA SCINCIDAE }\end{array}$ & Sg & $\mathrm{X}$ & $\mathrm{X}$ & $\operatorname{Pr}$ & $\mathrm{LC}$ & & $\mathrm{M}$ \\
\hline Scincella assata (Cope, 1864) & \multicolumn{7}{|c|}{ FAMILIA SCINCIDAE } \\
\hline \multicolumn{8}{|l|}{ FAMILIA TEIIDAE } \\
\hline Aspidoscelis deppii (Wiegmann, 1834) & Ad & $\mathrm{X}$ & $\mathrm{X}$ & & & & B \\
\hline Holcosus parvus (Barbour \& Noble, 1915) & Hр & $\mathrm{X}$ & $\mathrm{X}$ & & & & $\mathrm{M}$ \\
\hline \multicolumn{8}{|l|}{ FAMILIA XANTUSIIDAE } \\
\hline Lepidophyma smithii Bocourt, 1876 & Ls & & $\mathrm{X}$ & $\operatorname{Pr}$ & & & B \\
\hline \multicolumn{8}{|l|}{ SUBORDEN SERPENTES } \\
\hline \multicolumn{8}{|l|}{ FAMILIA BOIDAE } \\
\hline Boa imperator Daudin, 1803 & $\mathrm{Bi}$ & & $\mathrm{X}$ & A & & II & M \\
\hline \multicolumn{8}{|l|}{ FAMILIA COLUBRIDAE } \\
\hline Coluber mentovarius (Duméril, Bibron \& Duméril, 1854) & $\mathrm{Cm}$ & $\mathrm{X}$ & $\mathrm{X}$ & A & LC & & B \\
\hline Drymarchon melanurus (Duméril, Bibron \& Duméril, 1854) & $\mathrm{Dm}$ & $\mathrm{X}$ & & & $\mathrm{LC}$ & & B \\
\hline Drymobius margaritiferus (Schlegel, 1837) & Dma & $\mathrm{X}$ & $\mathrm{X}$ & & & & B \\
\hline Lampropeltis abnorma (Bocourt, 1886) & La & $\mathrm{X}$ & $\mathrm{X}$ & A & & & B \\
\hline Mastigodryas melanolomus (Cope, 1868) & Mme & $\mathrm{X}$ & & & LC & & B \\
\hline Oxybelis aeneus (Wagler, 1824) & $\mathrm{Oa}$ & & $\mathrm{X}$ & & & & B \\
\hline Oxybelis fulgidus (Daudin, 1803) & Of & & $\mathrm{X}$ & & & & B \\
\hline Pituophis lineaticollis (Cope, 1861) & $\mathrm{Pl}$ & & $\mathrm{X}$ & & LC & & B \\
\hline Spilotes pullatus Linnaeus, 1758 & Sp & $\mathrm{X}$ & $\mathrm{X}$ & & & & $\mathrm{B}$ \\
\hline Senticolis triaspis (Cope, 1866) & St & & $\mathrm{X}$ & & $\mathrm{LC}$ & & B \\
\hline Stenorrhina freminvillei Peters \& Orejasmiranda, 1970 & Sf & $\mathrm{X}$ & $\mathrm{X}$ & & & & B \\
\hline Tantilla rubra Cope, 1876 & $\operatorname{Tr}$ & $\mathrm{X}$ & $\mathrm{X}$ & $\operatorname{Pr}$ & $\mathrm{LC}$ & & B \\
\hline Trimorphodon biscutatus (Duméril, Bibron \& Duméril, 1854) & $\mathrm{Tb}$ & $\mathrm{X}$ & $\mathrm{X}$ & & & & B \\
\hline \multicolumn{8}{|l|}{ FAMILIA DIPSADIDAE } \\
\hline Conophis sp. & Csp & $\mathrm{X}$ & & & & & \\
\hline Coniophanes fissidens (Günther, 1858) & $\mathrm{Cf}$ & & $\mathrm{X}$ & & & & B \\
\hline Imantodes gemmistratus (Cope, 1861) & Ig & $\mathrm{X}$ & $\mathrm{X}$ & $\operatorname{Pr}$ & & & $\mathrm{B}$ \\
\hline Leptodeira maculata (Hallowell, 1861) & $\mathrm{Lm}$ & $\mathrm{X}$ & $\mathrm{X}$ & $\operatorname{Pr}$ & LC & & B \\
\hline Leptodeira nigrofasciata Günther, 1868 & Ln & & $\mathrm{X}$ & & $\mathrm{LC}$ & & B \\
\hline Leptodeira septentrionalis (Kennicott, 1859) & Lse & & $\mathrm{X}$ & & & & B \\
\hline Rhadinella godmani (Günther, 1865) & $\mathrm{Rg}$ & & $\mathrm{X}$ & & $\mathrm{LC}$ & & $\mathrm{M}$ \\
\hline Tropidodipsas fasciata Günther, 1858 & $\mathrm{Tf}$ & $\mathrm{X}$ & $\mathrm{X}$ & & & & $\mathrm{M}$ \\
\hline \multicolumn{8}{|l|}{ FAMILIA ELAPIDAE } \\
\hline Micrurus latifasciatus Schmidt, 1933 & $\mathrm{Ml}$ & $\mathrm{X}$ & $\mathrm{X}$ & & $\mathrm{LC}$ & & M \\
\hline Micrurus nigrocinctus (Girard, 1854) & $\mathrm{Mn}$ & & $\mathrm{X}$ & $\operatorname{Pr}$ & & & M \\
\hline \multicolumn{8}{|l|}{ FAMILIA LEPTOTYPHLODIDAE } \\
\hline Epictia phenops (Cope, 1875) & Epp & $\mathrm{X}$ & $\mathrm{X}$ & & & & B \\
\hline \multicolumn{8}{|l|}{ FAMILIA SIBYNOPHIIDAE } \\
\hline Scaphiodontophis annulatus (Duméril, Bibron \& Duméril, 1854) & San & & $\mathrm{X}$ & & & & M \\
\hline \multicolumn{8}{|l|}{ FAMILIA VIPERIDAE } \\
\hline Agkistrodon bilineatus Günther, 1863 & $\mathrm{Ab}$ & $\mathrm{X}$ & & $\operatorname{Pr}$ & NT & & M \\
\hline Crotalus simus Latreille, 1801 & Csi & $\mathrm{X}$ & $\mathrm{X}$ & & LC & & M \\
\hline
\end{tabular}


López-Vila et al.: Herpetofauna del sitio arqueológico Iglesia Vieja, Chis.

\begin{tabular}{|c|c|c|c|c|c|c|c|}
\hline Taxa & 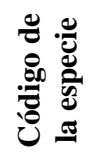 & 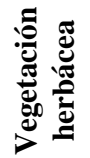 & 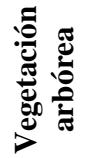 & 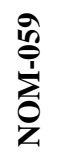 & Z & שِ & $\sum_{i=1}^{\infty}$ \\
\hline Porthidium dunni (Hartweg \& Oliver, 1938) & $\mathrm{Pd}$ & & $\mathrm{X}$ & $\mathrm{A}$ & LC & & $\mathrm{A}$ \\
\hline
\end{tabular}

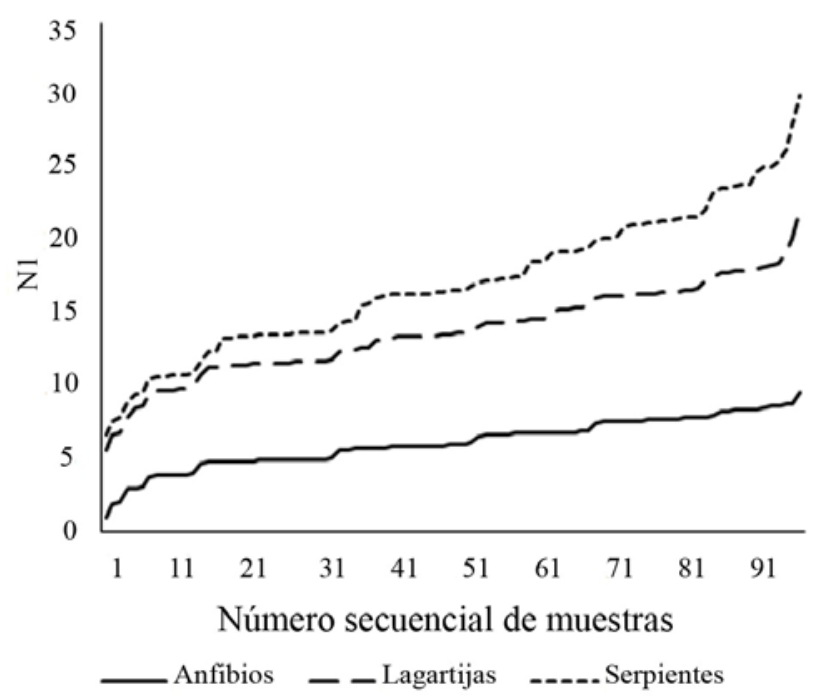

Figura 3. Curvas de diversidad acumulada expresada como números de primer orden $\left(\mathrm{N}_{1}\right.$ en la serie de Hill) para los grupos de anfibios, lagartijas y serpientes en el sitio arqueológico Iglesia Vieja.

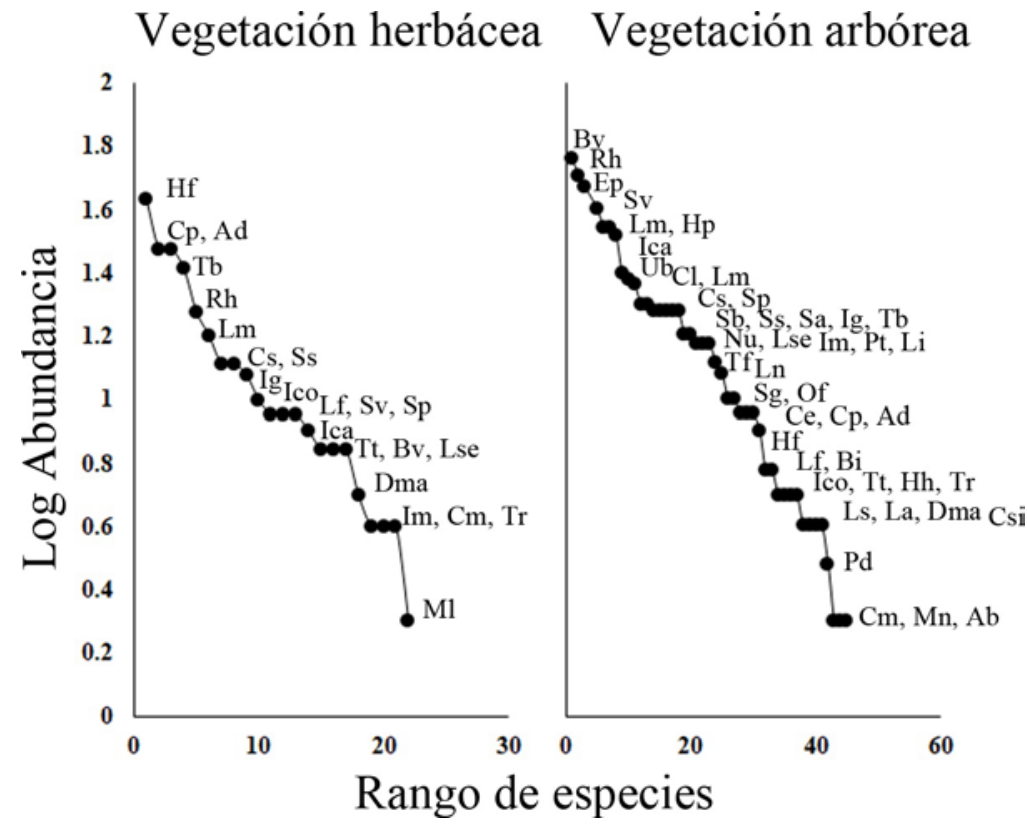

Figura 4. Diagramas de rangos de abundancia de especies de anfibios y reptiles para Vegetación herbácea y arbórea en el sitio arqueológico Iglesia Vieja. La clave de cada especie se presenta en el Cuadro 1. 


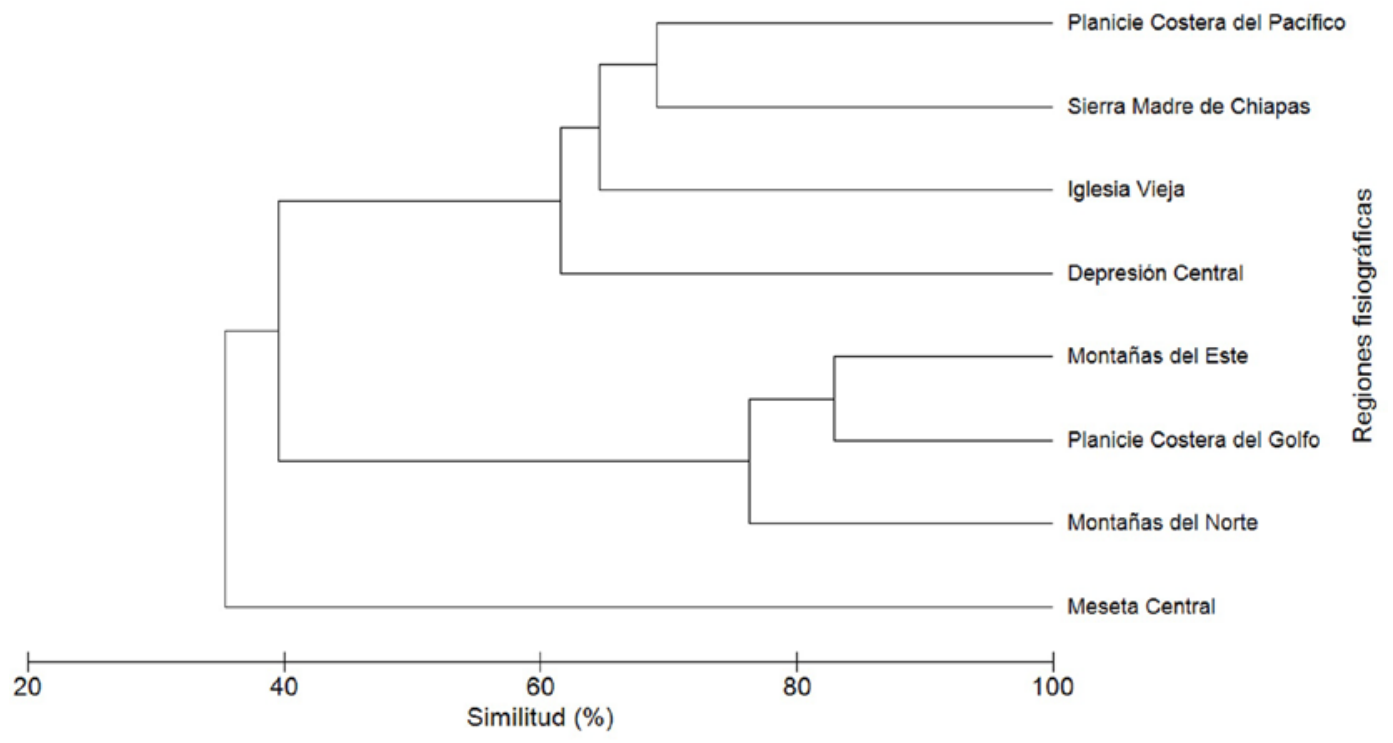

Figura 5. Dendrograma de similitud entre siete regiones fisiográficas de Chiapas y el sitio arqueológico Iglesia Vieja basada en datos de presencia y ausencia de especies.

Para el grupo de los anfibios, los estimadores Jackknife 1 y Bootstrap predicen entre 14 y 13 especies respectivamente, lo que muestra que se registró entre el $85.7 \%$ y el $92.3 \%$ de la riqueza esperada. En el caso de las lagartijas se estimaron 23 especies con Jackknife 1 y 21 especies con Boostrap, lo cual representa entre un $87 \%$ y $95 \%$ de la riqueza esperada de acuerdo a ambos estimadores, mientras que para el grupo de las serpientes, Jackknife 1 y Bootstrap predijeron entre 29 y 28 especies potencialmente presentes, registrándose entre $89.7 \%$ y $92.9 \%$ de la riqueza esperada (Fig. 2). El número de especies observadas de anfibios (12 spp.), lagartijas (20) y serpientes (29) no fue distinto al obtenido extrapolando al doble y triple el esfuerzo de muestreo.

En el caso de la diversidad ( $\mathrm{N}_{1}$ de Hill) acumulada no se observa una marcada asíntota para los tres grupos de herpetozoos (Fig. 3). Los diagramas de distribución de abundancia mostraron diferencias en la composición de especies por cobertura vegetal $(\mathrm{W}=406, \mathrm{p}<0.0001)$. En áreas con ausencia de cobertura arbórea se observó una mayor dominancia de especies que pueden ser favorecidas por la perturbación antrópica, como es el caso de la especie exótica Hemidactylus frenatus. Otras especies dominantes en alguna de las dos coberturas evaluadas son de hábitos generalistas, como Rhinella horribilis, Basiliscus vittatus o Aspidoscelis deppii (Fig. 4). Por otro lado, las especies menos dominantes de acuerdo con este muestreo fueron Dermophis mexicanus, Stenorrhina freminvillei y Epictia phenops.

De las 61 especies reportadas para el SAIV, tres especies se encuentran en el apéndice II de la CITES (Boa imperator, Heloderma horridum e Iguana iguana); tres especies mostraron ser altamente vulnerables de acuerdo al EVS (Ctenosaura pectinata, Heloderma horridum y Porthidium dunni); 21 estuvieron en alguna categoría de riesgo de la NOM-059-SEMARNAT y 36 en la lista roja de la IUCN (Cuadro 1). Finalmente, al comparar la herpetofauna del área de estudio con la reportada para las siete regiones fisiográficas de Chiapas, el SAIV tuvo mayor afinidad herpetofaunística con las regiones Planicie Costera del Pacífico (66.53\%) y Sierra Madre de Chiapas (62.34 \%). Las unidades regionales para las siete provincias fisiográficas y el SAIV formadas en el dendrograma se muestran en la figura 5. 
López-Vila et al.: Herpetofauna del sitio arqueológico Iglesia Vieja, Chis.

\section{DISCUSIÓN}

En Chiapas se han reportado 330 especies de herpetozoos (Johnson et al., 2015a), por lo que los anfibios y reptiles registrados en el SAIV representan al 18.48\% de la herpetofauna estatal. Aunque el área de influencia del SAIV es de tan solo 60 ha, la densidad especies en nuestra área de estudio fue mayor a la reportada en otras zonas de un área mucho mayor y que contemplan selva baja, tal es el caso del Cerro de Guiengola, Oaxaca (Martín-Regalado et al., 2011) y el Corredor Puerto Madero, Chiapas (Luna-Reyes et al., 2012).

En lo que respecta a las regiones fisiográficas colindantes con el área de estudio, el $46.9 \%$ y el 32.7\% de las especies de la Planicie Costera de Chiapas y la Sierra Madre, respectivamente, se encuentran alojadas en esta área. Además de las regiones fisiográficas, el SAIV tiene colindancia al norte con la Reserva de la Biosfera la Sepultura (REBISE), la cual cuenta con un catálogo herpetofaunístico de 73 especies (24 de anfibios y 49 de reptiles) (INE, 1999). Treinta y dos de estas especies se registraron en Iglesia Vieja (45.2\%), de las cuales siete fueron de anfibios y 25 de reptiles.

Los valores de riqueza estimada indicaron niveles adecuados en el esfuerzo de muestreo aquí propuesto para los tres grupos de herpetozoos, alcanzando estimaciones superiores al 85\% de la completitud del inventario; esto considerando que se ha propuesto un $80 \%$ de representatividad mínima para efectuar comparaciones válidas entre hábitats, temporadas o inventarios (Colwell \& Coddington, 1995; Pineda \& Halffter, 2004). Sin embargo, para el caso de los anfibios, la riqueza representada en la curva de acumulación no alcanza una marcada asíntota, siendo esta tendencia observada en el análisis de diversidad acumulada para los tres grupos. En este contexto, el uso de los números de la serie de Hill en curvas acumuladas ofrecen una mayor certidumbre en estimaciones de la diversidad, controlando eficazmente la contribución de los taxones raros a la muestra (Kang et al., 2016). Esto permite detectar interpretaciones erróneas en las estimaciones tradicionales de riqueza, las cuales pueden depender en gran medida al protocolo de muestreo utilizado (Moreno et al., 2011).

La riqueza de anfibios tiende a ser comparativamente menor a la de los reptiles en ambientes con definida estacionalidad climática, como lo es la planicie costera del Pacífico mexicano. Las especies de este último grupo pueden presentar una distribución más homogénea durante el ciclo lluvias-estiaje (García \& Cabrera-Reyes, 2008; Leyte-Manrique et al., 2016), debido principalmente a las ventajas adaptativas de muchos reptiles para tolerar eficazmente las variaciones en temperatura y precipitación. Para el caso de los anfibios, su presencia y abundancia tiende a asociarse a la disponibilidad de cuerpos de agua perennes o a la estación lluviosa; a excepción de pocas especies con un modo reproductivo que les permita cierta independencia del medio acuático, como ocurre con Craugastor loki, la cual es observable durante todo el año en el SAIV. La identificación de ambientes acuáticos efímeros en el SAIV y un mayor esfuerzo de muestreo en estas áreas, probablemente permitirá incrementar el registro de nuevas especies para el sitio. No obstante la tendencia en selvas secas es la de encontrar una menor riqueza de anfibios (Martín-Regalado et al., 2011).

Con base en el tipo de cobertura vegetal, el registro aquí de un mayor número de especies en el hábitat arbóreo (56 spp.) que en el herbáceo (43), es una tendencia descrita también en otros estudios. Luna-Reyes et al. (2012) encontraron que aunque más del 66\% de las especies de herpetozoos se comparten entre pastizales y ambientes arbóreos (manglares y selvas) de la costa de Chiapas, existen marcadas diferencias en cuanto a diversidad, la cual es notablemente mayor en estos últimos. MoralesMávil et al. (2001) reportan que para un área de borde de selva alta en Veracruz, el 47.6\% de especies de reptiles ocurren tanto en hábitats arbóreos como en pastizales. Por otro lado, se ha observado que la disimilitud en la herpetofauna entre bosques de origen primario y secundario tiende a ser menor (UrbinaCardona \& Londoño-Murcia, 2003). De acuerdo con la heterogeneidad ambiental, los hábitats que estructuralmente son más complejos frecuentemente exhibirán una mayor riqueza y diversidad de especies 
(Stewart et al., 2000). Algunos autores consideran que la vegetación en muchos casos no contribuye a explicar la distribución y estructura de las comunidades herpetológicas, debido principalmente a los patrones de uso de hábitat de las especies (García \& Ceballos, 1994). Sin embargo el tipo de cobertura vegetal si suele estar relacionado con la diversidad de microhábitats que definen la abundancia de organismos con hábitos acuáticos, terrestres, fosoriales o arborícolas (Whitfield \& Pierce, 2005; Wells, 2007; Calderón-Mandujano et al., 2008). Del total de las especies reportadas en este trabajo, el 35\% se encontró en alguna categoría de riesgo de la NOM-059-SEMARNAT, de las cuales una ocurre en todas las categorías de riesgo consideradas en este escrito: Heloderma horridum (Amenazada en la NOM, Least Concern en la IUCN, en el apéndice II del CITES y una puntuación Alta en el Environmental Vulnerability Score). Catalogadas en tres categorías le siguen Dermophis mexicanus, Iguana iguana, Boa imperator, Agkistrodon bilineatus y Porthidium dunni; todas ellas con evaluaciones de riesgo media a alta.

En lo que concierne a ciertas especies del SAIV, la captura de un organismo del género Conophis no pudo concretarse en este muestreo, por lo que la identificación se realizó con los rasgos observados pudiéndose tratar, de acuerdo a su distribución y características morfológicas, de Conophis vittatus. Otra especie detectada no corroborada, pero con potencial de distribución en el sitio, es Scinax staufferi, un anfibio de la familia Hylidae ya registrado a baja altitud en la Planicie Costera del Pacífico (Luna-Reyes et al., 2012).

Los patrones de similitud herpetofaunística de Chiapas explorados por Johnson et al. (2015a) mostraron que la Planicie Costera del Pacífico, la Sierra Madre y la Depresión Central, integran una misma unidad zoogeográfica. De acuerdo a nuestro estudio, el patrón de similitud del SAIV con respecto al grupo detectado por Johnson et al. (2015a) correspondería a la influencia de los ambientes subhúmedos predominantes en las tres regiones fisiográficas mencionadas. La localización del SAIV en las estribaciones de la Sierra Madre, podría ubicarla como un corredor subhúmedo para las especies entre la Planicie Costera y la Depresión Central.

La herpetofauna del SAIV presentó una riqueza de especies de selvas secas relativamente alta, considerando su reducido tamaño y fragmentación del hábitat, albergando además a varias especies catalogadas bajo alguna categoría de riesgo a nivel nacional e internacional. El presente trabajo constituye la primera línea base referente a estudios herpetológicos, y en general el primero sobre biodiversidad, en este sitio. A fin de establecer criterios para el manejo de un sitio arqueológico y sus ecosistemas naturales, es altamente recomendable continuar con investigaciones sobre la estructura de la comunidad y dinámica de sus poblaciones para este y otros grupos bióticos prioritarios.

AgRAdecimientos. Los autores agradecemos al Dr. Uri Omar García Vázquez por revisar y corroborar los registros aquí presentados; a Jesús Ernesto Pérez Sánchez por su apoyo en campo y al personal del SAIV: Guillermo Salazar, José del Carmen y Tadeo por las facilidades brindadas para llevar a cabo este estudio.

\section{LITERATURA CITADA}

Báez, J. C., Camiñas, J. A., Sacchi, J. (2016) Reptile diversity associated to archaelogical sites: the significance of ancient ruins for reptile conservation. Boletín de la Asociación Herpetológica Española, 27, 101-103.

Calderón-Mandujano, R. R., Galindo-Leal, C., Cedeño-Vázquez, R. (2008) Utilización de hábitat por reptiles en estados sucesionales de selvas tropicales de Campeche, México. Acta Zoológica Mexicana (nueva serie), 24, 95-114. 
Casazza, G., Zappa, E., Mariotti, G., Médail, F., Minuto, L. (2008) Ecological and historical factors affecting distribution pattern and richness of endemic plant species: the case of the Maritime and Ligurian Alps hotspot. Diversity and Distributions, 14, 47-58.

Clarke, K. R., Gorley, R. N. (2006) PRIMER V6: User Manual/Tutorial. PRIMER-E, Plymouth.

Colwell, R. K., Coddington, J. A. (1995). Estimating terrestrial biodiversity through extrapolation. Pp. 101-118. In: D. L. Hawksworth, (Ed). Biodiversity Measurement and Estimation. Chapman \& Hall, New York.

Colwell, R. K., Mao, C. X., Chang, J. (2005) Interpolando, extrapolando y comparando las curvas de acumulación de especies basadas en su incidencia. Pp. 73-84. In: G. Halffter, J. Soberón, P. Koleff and A. Melic, (Eds). Sobre Diversidad Biológica: El significado de las diversidades alfa, beta y gamma. CONABIO, SEA, Grupo Diversitas-México, CONACYT, Zaragoza, España.

CONANP. (2010a) Programa de conservación y manejo Monumento Natural Yaxchilán. SEMARNATCONANP, México, D.F.

CONANP. (2010b) Programa de conservación y manejo Monumento Natural Bonampak. SEMARNATCONANP, México, D.F.

Ferreira-García, M. E., Canseco-Márquez, L. (2006) Estudio de la herpetofauna del monumento natural de Yaxchilán, Chiapas, México. Pp. 293-310. In: A. Ramírez-Bautista, L. CansecoMárquez and F. Mendoza-Quijano (Eds). Inventarios herpetofaunísticos de México: avances en el conocimiento de su biodiversidad. Publicaciones especiales de la Sociedad Herpetológica Mexicana, México.

Flores-Villela, O., Mendoza, F. Q., González, G. P. (1995) Recopilación de claves para la determinación de Anfibios y Reptiles de México. Publicaciones Especiales del Museo de Zoología “Alfonso L. Herrera” No. 10. Universidad Nacional Autónoma de México, México D.F.

García, A., Cabrera-Reyes, A. (2008) Estacionalidad y estructura de la vegetación en la comunidad de anfibios y reptiles de Chamela, Jalisco, México. Acta Zoológica Mexicana (nueva serie), 24, 91-115.

García, A., Ceballos, G. (1994) Guía de campo de los reptiles y anfibios de la costa de Jalisco, México. Fundación Ecológica Cuixmala /UNAM, México. D.F.

Hammer, Ø., Harper, D. A. T., Ryan, P. D. (2001) PAST: Paleontological Statistics software package for education and data analysis. Palaeontologia Electrónica, 4, 9.

Hausdorf, B. (2002) Units in biogeography. Systematic Biology, 51, 648-652.

Heyer, R. W., Donelly, M. A., McDiarmid, R. W., Hayek, L., Foster, M. S. (1994) Measuring and monitoring biological diversity: Standard methods for amphibians. Smithsonian Institution Press, Washington, D.C.

INAH (Instituto Nacional de Antropología e Historia) (2017) Red de zonas arqueológicas. Disponible en: http://inah.gob.mx/images/zonas/lista/pagina.html (acceso en mayo 2017).

INE (Instituto Nacional de Ecología) (1999) Programa de manejo de la Reserva de la Biósfera La Sepultura. Instituto Nacional de Ecología-SEMARNAT, México, D.F., 247 pp.

INEGI (Instituto Nacional de Estadística y Geografía) (2014) Marco Geoestadístico Municipal. V. 6.2. Disponible en: http://www.inegi.org.mx/geo/contenidos/geoestadistica/ (acceso abril 2017)

IUCN. (2015) The IUCN Red List Threatened Species. Version 2015-4. Disponible en: http://www.iucnredlist.org (acceso abril 2016)

Johnson, J. D. (1989) A biogeographic analysis of the herpetofauna of northwestern Nuclear Central America. Milwaukee Public Museum Contributions in Biology and Geology, 76, 1-66.

Johnson, J. D., Mata-Silva, V., García-Padilla, E., Wilson, L. D. (2015a) The herpetofauna of Chiapas, Mexico: composition, physiographic distribution, and conservation status. Mesoamerican Herpetology, 2, 272-329.

Johnson, J. D., Mata-Silva, V., Wilson, L. D. (2015b) A conservation reassessment of the Central American herpetofauna based on the EVS measure. Amphibian \& Reptile Conservation, 9, 1-94.

Jost, L. (2006) Entropy and diversity. Oikos, 113, 363-375. 
Kaneko, A. (2006) Iglesia Vieja. Pp. 345-366. In: D. Aramoni-Calderón, T. A. Lee-Whiting and M. Lisbona-Guillén (Coord). Presencia Zoque. Una aproximación multidisciplinaria. UNICACH, COCYTECH, UNACH, UNAM. Tuxtla Gutiérrez, México.

Kaneko, A. (2011) Iglesia Vieja: Un sitio megalítico del Clásico Temprano en la costa del Pacífico de Chiapas. Pp. 663-680. In: B. Arroyo, L. Paiz, A. Linares \& A. Arroyave (Eds). XXIV Simposio de Investigaciones arqueológicas en Guatemala. Museo Nacional de Arqueología y Etnología, Guatemala.

Kang, S., Rodrigues, J. L. M., Ng, J. P., Gentry, T. J. (2016) Hill number as a bacterial diversity measure framework with high-throughput sequence data. Scientific Reports, 6, 1-4.

Kelly-Hernández. A., Lara-Hernández. F. A., Servín-Torres, J. L., León-López, E., Peña-Ramírez, J. R., Gheno-Heredia, J. (2014) Rescate de herpetofauna por el impacto de la restauración de la zona arqueológica de Toxpan-USBI UV, en la ciudad de Córdoba, Veracruz. Pp. 58. In: Sociedad Herpetológica Mexicana (Ed). Memorias de la XIII Reunión Nacional de Herpetología. SHM, CONABIO, CONAN, Conservación de la Biodiversidad del Centro de México A.C., Universidad Autónoma de Aguascalientes, Aguascalientes.

Köhler, G. (2008) Reptiles of Central America. Herpeton, Offenbach, Alemania.

Köhler, G. (2011) Amphibians of Central America. Herpeton, Offenbach, Alemania.

Lee, J. C. (1996) The Amphibians and Reptiles of the Yucatan Peninsula. Cornell University Press, New York.

Leyte-Manrique, A., Morales-Castorena, J. P., Escobedo-Morales, L. A. (2016) Variación estacional de la herpetofauna en el cerro del Veinte, Irapuato, Guanajuato, México. Revista Mexicana de Biodiversidad, 87, 150-155.

Lips, K. R., Reaser, J. K., Young, B. E., Ibañez, R. (2001) Amphibian monitoring in Latin America: a protocol manual. Society for the Study of Amphibians and Reptiles Oxford, Ohio.

Luna-Reyes, R., Vidal-López, R., Hernández-García, E., Montesino-Castillejos, H. (2012) Anfibios y reptiles de la región marina prioritaria Corredor Puerto Madero, Chiapas, México. Pp. 280-230. In: Sánchez, A.J., Chiappa-Carrara, X. \& Brito-Pérez, R. (Eds.), Recursos acuáticos costeros del sureste. RECORECOS/UNAM, Mérida, México.

Magurran, A. E. (2004) Measuring Biological Diversity. Blackwell Science, Malden, Massachussets.

Márquez-Rodríguez, J. (2014) La importancia de los refugios de Timon lepidus en los yacimientos arqueológicos. Boletín de las Asociación Herpetológica Española, 25, 85-87.

Martín-Regalado, C. N., Gómez-Ugalde, R. M., Cisneros-Palacios, M. E. (2011) Herpetofauna del cerro Guiengola, Istmo de Tehuantepec, Oaxaca. Acta Zoológica Mexicana (nueva serie), 27, 359-376.

McGill, B. J., Etienne, R. S., Gray, J. S., Alonso, D., Anderson, M. J., Benecha, H. K., Dornelas, M., Enquist, B. J., Green, J. L., He, F., Hurlbert, A. H., Magurran, A. E., Marquet, P. A., Maurer, B. A., Ostling, A., Soykan, C. U., Ugland, K. I., White, E. P. (2007) Species abundance distributions: moving beyond single prediction theories to integration within an ecological framework. Ecological Letters, 10, 995-1015.

Meave del Castillo, J. A. (2000) Caracterización biológica del Monumento Natural Yaxchilán como un elemento fundamental para el diseño de su plan rector de manejo. Informe final SNIB-CONABIO proyecto No. M099. Universidad Nacional Autónoma de México/CONABIO, México D. F.

Mireles-Merchant, R., Bustos-Zagal, M. G. (2014) Diversidad, distribución y amenazas de la herpetofauna de Xochicalco, Morelos. Pp. 152. In: Sociedad Herpetológica Mexicana (Ed). Memorias de la XIII Reunión Nacional de Herpetología. SHM, CONABIO, CONAN, Conservación de la Biodiversidad del Centro de México A.C., Universidad Autónoma de Aguascalientes, Aguascalientes.

Morales-Mávil, J., Hernández-González, M., Bravo-Méndez, O. (2001) Biomasa de reptiles en un borde de selva de la región de Los Tuxtlas, Veracruz, México. Foresta Veracruzana, 3, 25-30.

Moreno, C. E. (2001) Métodos para medir la biodiversidad. M \& T SEA Vol. 1., CYTED/ORCITUNESCO/SEA, Zaragoza, España. 
López-Vila et al.: Herpetofauna del sitio arqueológico Iglesia Vieja, Chis.

Moreno, C. E., Barragán, F., Pineda, E., Pavón, N. P. (2011) Re-análisis de la diversidad alfa: alternativas para interpretar y comparar información sobre comunidades ecológicas. Revista Mexicana de Biodiversidad, 82, 1240-1261.

Olivera, D., Castillo, L., Gutiérrez, G. (2016) Primer registro de Phyllodactylus sentosus (Squamata: Phyllodactylidae) para el valle del río Chillón, Lima, Perú. Revista Peruana de Biología, 23, 321-324.

Pennington, T. D., Sarukhán, J. (2005) Árboles tropicales de México. Manual para la identificación de las principales especies. UNAM, FCE, México, D.F.

Percino-Daniel, R., Cruz-Ocaña, E., Pozo-Ventura, W., Velázquez-Velázquez, E. (2013) Diversidad de reptiles en dos microcuencas del río Grijalva, Chiapas, México. Revista Mexicana de Biodiversidad, 84, 938-948.

Pineda, E., Halffter, G. (2004) Species diversity and habitat fragmentation: frogs in a tropical montane landscape in Mexico. Biological Conservation, 117, 499-508.

SEMARNAT. (2010) Norma Oficial Mexicana NOM-059-SEMARNAT-2010. Protección ambientalEspecies nativas de México de flora y fauna silvestres-Categorías de riesgo y especificaciones para su inclusión, exclusión o cambio-Lista de especies en riesgo. Diario Oficial de la Federación30 diciembre, segunda sección, 1-77, México.

Sokal, R. R., Rohlf, F. J. (1981) Biometry. W. H. Freeman, Nueva York.

Somaweera, R., Ukuwela, K., Karunaratne, S. (2001) Menikdena: a Local Herptofauna Hotspot. Occasional papers of the Amphibia \& Reptile Research Organization of Sri Lanka. 2, 1-8.

Stewart, A. J. A., John, E. A., Hutchings, M. J. (2000) The world is heterogeneous: ecological consequences of living in a patchy environment. Pp. 1-8. In: Hutchings, M. J., John, E. A. and Stewart, A. J. A. (Eds). The Ecological Consequences of Environmental Heterogeneity. Blackwell, United Kingdom.

Urbina-Cardona, J. N., Londoño-Murcia, M. C. (2003) Distribución de la comunidad de herpetofauna asociada a cuatro áreas con diferente grado de perturbación en la Isla Gorgona, Pacífico Colombiano. Revista de la Academia de Ciencias Exactas, Físicas y Naturales, 27, 105-113.

Wells, K. D. (2007) The Ecology and Behavior of Amphibians. The University of Chicago Press, Chicago.

Whitfield, S. M., Pierce, M. S. F. (2005) Tree buttress microhabitat use by a Neotropical leaf-litter herpetofauna. Journal of Herpetology, 39, 192-198.

Wilson, L. D., Mata-Silva, V., Johson, J. D. (2013a) A conservation reassessment of the reptiles of Mexico based on the EVS measure. Amphibian \& Reptile Conservation, 7, 1-47.

Wilson, L. D., Johson, J. D., Mata-Silva, V. (2013b) A conservation reassessment of the amphibians of Mexico based on the EVS measure. Amphibian \& Reptile Conservation, 7, 97-127. 